\title{
Reduced-Complexity Soft-Decision Aided PSK Detection
}

\author{
Chao Xu, Dandan Liang, Shinya Sugiura*, Soon Xin Ng and Lajos Hanzo \\ School of ECS, University of Southampton, SO17 1BJ, United Kingdom. \\ Email: $\{$ cx1g08,dl4e08,sxn,lh\}@ecs.soton.ac.uk, http://www-mobile.ecs.soton.ac.uk
}

\begin{abstract}
In this paper, we propose to reduce the complexity of both the Approx-Log-MAP algorithm as well as of the MaxLog-MAP algorithm, which were designed for soft-decision aided PSK detectors. First of all, we extend the shown a posteriori PSK symbol probability formula and streamline it by eliminating its unnecessary calculations in the context of the Approx-LogMAP algorithm. Secondly, we reduce the complexity of the MaxLog-MAP algorithm, where the maximum a posteriori symbol probability may be obtained without evaluating and comparing all the candidate symbol probabilities. Furthermore, we apply our new soft detection arrangement to a variety of coded systems. Our simulation results demonstrate that a significant detection complexity reduction was achieved by our design without any performance loss. For example, a factor two complexity reduction was achieved by the proposed Max-Log-MAP algorithm, when it was invoked for detecting QPSK symbols, which is expected to be significantly higher, when invoked for 16QAM.
\end{abstract}

\section{INTRODUCTION}

Channel coding is an essential component of virtually all communication systems. The classic BCJR algorithm of [1] invoked the MAP algorithm for channel decoding. Following the conception of the Soft Output Viterbi Algorithm (SOVA) [2] for reducing the complexity of the MAP algorithm, substantial research efforts have been dedicated to channel codes. An outstanding invention was the Log-MAP algorithm [3], which operates the MAP algorithm in the logarithm domain. The sub-optimal Max-Log-MAP algorithm was also proposed in [3], which searched for the two maximum a posteriori symbol probabilities having their specific bit fixed to 1 and 0 , respectively. In order to compensate for the sub-optimal Max-Log-MAP algorithm, the so-called Approx-Log-MAP algorithm was proposed in [4], which relied on a look-up table in order to take into account the differences between the candidates. Since then, the Approx-Log-MAP algorithm and the Max-Log-MAP algorithm have been widely used for soft-decision aided detection schemes.

The significant technical breakthrough of Turbo Codes (TCs) was proposed in [5], where a substantial performance improvement was achieved by exchanging extrinsic information between two Recursive Systematic Convolutional (RSC) decoders. Since then, the appealing iterative decoding of concatenated codes has inspired numerous researchers to aim for achieving a near-capacity performance in diverse system

The financial support of the RC-UK under the auspices of the India-UK Advanced Technology Centre (IU-ATC) and that of the EPSRC under the China-UK science bridge as well as that of the EU's Concerto project is gratefully acknowledged.

Shinya Sugiura is currently with the Toyota Central R\&D Labs, Inc., Aichi 480-1192, Japan (e-mail:sugiura@ieee.org). contexts [6], [7], [8], [9]. In [10], the PSK demodulator was modified to be able to process soft bits, so that exchanging extrinsic information may be carried out between the outer channel decoder and the inner PSK demodulator. Moreover, the EXtrinsic Information Transfer (EXIT) charts was proposed in [11] for analyzing the convergence behaviour of turbo detection. In order to eliminate the error floor often observed in two-component concatenated codes, it was proposed in [12] that a further Unity Rate Code (URC) may be incorporated, so that an infinitesimally low BER may be achieved by a threestage turbo detector. Furthermore, the IRregular Convolutional Code (IRCC) concept [13] was proposed to replace the regular convolutional codes, where the area between the EXIT curve of the outer channel code and that of the inner amalgamated URC and PSK block was minimized. As a result, a vanishingly low BER may be achieved at a near-capacity SNR [14], [6].

As researchers have inched closer and closer to the channel capacity, the complexity of the resultant communication systems was increased. The soft-decision aided PSK demodulators typically contribute a large portion of the turbo detection complexity. Therefore, in this paper, we revisit the design of soft-decision aided PSK demodulators and seek to reduce their decoding complexity. Against this background, the novel contributions of this paper are as follows:

1) It is widely recognized that the hard-decision aided PSK demodulators make their decisions on a bit-bybit basis. For instance, only either the real part or the imaginary part of the received QPSK symbol is of interest for making a hard bit decision. By contrast, the soft-decision aided PSK demodulators are symbolby-symbol based [10], [7], i.e. all the candidate PSK symbol probabilities have to be evaluated and compared for producing a soft bit output. However, we observe that the Max-Log-MAP algorithm only has to get the maximum symbol probabilities, which is similar to the action of hard PSK detectors, when searching for their minimum metrics. Therefore, we propose to operate the Max-Log-MAP algorithm on a bit-by-bit basis, where not all the candidate symbol probabilities have to be calculated for producing a soft bit output.

2) Since the Approx-Log-MAP algorithm is designed to compensate for the differences between the candidate a posteriori symbol probabilities, it has to be operated on a symbol-by-symbol basis. To mitigate its complexity, we extend the equations used for evaluating the a posteriori PSK symbol probability, where the unneccessary calculations are eliminated. 
The remainder of this paper is organized as follows. In Section II, we briefly review the bit-by-bit based hard PSK detection. The Log-MAP algorithm, the Approx-Log-MAP algorithm, as well as the Max-Log-MAP algorithm designed for soft PSK detection are detailed in Section III. In Sections IV and V, we propose to reduce the complexity of the Approx-Log-MAP algorithm and that of the Max-Log-MAP algorithm, respectively. Our performance results are provided in Section VI, while our conclusions are offered in Section VII.

\section{HARD-Decision Aided PSK Detection}

For a Single-Input Multiple-Output (SIMO) system, the signal received by $N$ receive antennas may be expressed as:

$$
\mathbf{Y}_{n}=s_{n} \mathbf{H}_{n}+V_{n},
$$

where $s_{n}$ denotes the $L$-PSK symbol, while the $(1 \times N)$ element vectors $\mathbf{Y}_{n}, \mathbf{H}_{n}$ and $\mathbf{V}_{n}$ refer to the received signal vector, the Rayleigh fading channel vector and the Additive White Gaussian Noise (AWGN) vector, which has a zero mean and a variance of $N_{0}$, respectively. Naturally, upon receiving $\mathbf{Y}_{n}$ in Eq. (1), the ML detection may be formulated as:

$$
\hat{s}^{l}=\min _{s^{l} \in \mathbf{S}}\left\|\mathbf{Y}_{n}-s^{l} \mathbf{H}_{n}\right\|^{2},
$$

where $\mathbf{S}$ indicates the $L$-PSK symbol set for $\left\{s^{l}\right\}_{l=1}^{L}$, while the coherent receiver assumes a perfect knowledge of $\mathbf{H}_{n}$. The Euclidean norm calculation of Eq. (2) leads to the decision variable of:

$$
z_{n}=\mathbf{Y}_{n} \mathbf{H}_{n}^{H}
$$

Therefore, the vector-by-vector based ML detection of Eq. (2) may be simplified to be operated on a symbol-by-symbol basis:

$$
\hat{s}^{l}=\min _{s^{l} \in \mathbf{S}}\left|z_{n}-s^{l}\right|^{2} .
$$

In order to show the equivalence between Eq. (2) and Eq. (4), we further extend the decision metric of Eq. (2) as:

$$
\begin{aligned}
& \left\|\mathbf{Y}_{n}-s^{l} \mathbf{H}_{n}\right\|^{2}=\operatorname{tr}\left[\left(\mathbf{Y}_{n}-s^{l} \mathbf{H}_{n}\right)\left(\mathbf{Y}_{n}-s^{l} \mathbf{H}_{n}\right)^{H}\right] \\
& =\left\|\mathbf{Y}_{n}\right\|^{2}+\left|s^{l}\right|^{2} \cdot\left\|\mathbf{H}_{n}\right\|^{2}-\left[\left(s^{l}\right)^{*} \mathbf{Y}_{n} \mathbf{H}_{n}^{H}+s^{l} \mathbf{H}_{n} \mathbf{Y}_{n}^{H}\right] \\
& =\left\|\mathbf{Y}_{n}\right\|^{2}+\left|s^{l}\right|^{2} \cdot\left\|\mathbf{H}_{n}\right\|^{2}-2 \operatorname{Re}\left\{\left(s^{l}\right)^{*} \mathbf{Y}_{n} \mathbf{H}_{n}^{H}\right\},
\end{aligned}
$$

while the decision metric of Eq. (4) may be extended as:

$$
\begin{aligned}
\left|z_{n}-s^{l}\right|^{2} & =\left|z_{n}\right|^{2}+\left|s^{l}\right|^{2}-\left[\left(s^{l}\right)^{*} z_{n}+s^{l} z_{n}^{*}\right] \\
& =\left|z_{n}\right|^{2}+\left|s^{l}\right|^{2}-2 \operatorname{Re}\left\{\left(s^{l}\right)^{*} z_{n}\right\} .
\end{aligned}
$$

Observe in Eq. (5) and Eq. (6) that $\left\|\mathbf{Y}_{n}\right\|^{2},\left|s^{l}\right|^{2},\left\|\mathbf{H}_{n}\right\|^{2}$ and $\left|z_{n}\right|^{2}$ are all invariant over the different candidates $s^{l}$. Therefore, minimizing $\left\|\mathbf{Y}_{n}-s^{l} \mathbf{H}_{n}\right\|^{2}$ and minimizing $\left|z_{n}-s^{l}\right|^{2}$ over all possible $s^{l}$ are equivalent.

The bit-by-bit based hard PSK detection may be further implemented according to the constellation diagram. Taking QPSK as an example, the hard-decision based on the constellation diagram of Fig. 1 is given by:

$$
\hat{b}_{1}=\left\{\begin{array}{ll}
1, & \operatorname{Im}\left(z_{n}\right)<0 \\
0, & \text { otherwise }
\end{array} \quad, \quad \hat{b}_{2}=\left\{\begin{array}{ll}
1, & \operatorname{Re}\left(z_{n}\right)<0 \\
0, & \text { otherwise }
\end{array} .\right.\right.
$$

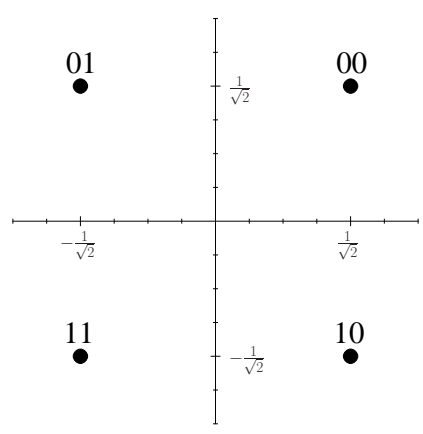

Fig. 1. Constellation diagram of QPSK, where the constellation diagram of any $L$-PSK scheme with $(L \geq 4)$ is anti-clockwisely rotated by $\pi / L$.

We note that we deliberately rotated all the constellations of $L$-PSK $(L \geq 4)$ in [8] anti-clockwise by a phase of $\frac{\pi}{L}$, so that there are exactly $L / 4$ constellation points in each quadrant. This feature will be beneficial for reducing the soft PSK detectors' complexity.

\section{Soft-Decision Aided PSK Detection}

For the sake of turbo detection, the hard-decision aided PSK detection should be modified to be able to both accept and to produce soft bit, which is in the form of Log Likelihood Ratios (LLRs). The a posteriori LLRs produced by the Log-MAP algorithm may be expressed as [3]:

$$
L_{p}\left(b_{k} \mid \mathbf{Y}\right)=\ln \left[\frac{\sum_{s^{l} \in \mathbf{S}_{b_{k}=1}} \exp \left(d_{l}\right)}{\sum_{s^{l} \in \mathbf{S}_{b_{k}=0}} \exp \left(d_{l}\right)}\right]=L_{a}\left(b_{k}\right)+L_{e}\left(b_{k}\right),
$$

where $\mathbf{S}_{b_{k}=1}$ and $\mathbf{S}_{b_{k}=0}$ refer to the symbol set for $\left\{s^{l}\right\}_{l=1}^{L}$, when the specific bit $b_{k}$ is fixed to 1 and 0 , respectively, while $L_{p}, L_{a}$ and $L_{e}$ denote the a posteriori, the a priori and the extrinsic LLR values, respectively. Given a specific $L$-PSK symbol $s^{l}$, its a posteriori symbol probability in Eq. (8) is defined as [10]:

$$
d_{l}=-\frac{\left|z_{n}-s^{l}\right|^{2}}{N_{0}}+\sum_{j=1}^{\log _{2} L} b_{j} L_{a}\left(b_{j}\right)
$$

The Log-MAP algorithm of Eq. (8) may be simplified by the so-called Approx-Log-MAP algorithm as [4]:

$$
L_{p}\left(b_{k} \mid \mathbf{Y}\right)=\mathrm{jac}_{s^{l} \in \mathbf{S}_{b_{k}=1}}\left(d_{l}\right)-\mathrm{jac}_{s^{l} \in \mathbf{S}_{b_{k}=0}}\left(d_{l}\right),
$$

where jac denotes the corrected Jacobian logarithm, which compensates the differences between the candidate a posteriori symbol probabilities according to a look-up table [4].

When designing a low-complexity soft detector, the socalled Max-Log-MAP algorithm may be invoked [3]:

$$
L_{p}\left(b_{k} \mid \mathbf{Y}\right)=\max _{s^{l} \in \mathbf{S}_{b_{k}}=1}\left(d_{l}\right)-\max _{s^{l} \in \mathbf{S}_{b_{k}}=0}\left(d_{l}\right) .
$$

Since only two maximum a posteriori symbol probabilities are taken into account in Eq. (11), the Max-Log-MAP algorithm imposes a slight performance degradation. 


\section{ReducED-COMPlexity Soft PSK DETECTION USING THE APPROX-LOG-MAP ALGORITHM}

Similar to our analysis presented in Eq. (6), the a posteriori symbol probability of Eq. (9) may be extended as:

$$
\begin{array}{r}
d_{l}=-\frac{\left|z_{n}\right|^{2}+\left|s^{l}\right|^{2}-2 \operatorname{Re}\left[\left(s^{l}\right)^{*} z_{n}\right]}{N_{0}}+\sum_{j=1}^{\log _{2} L} b_{j} L_{a}\left(b_{j}\right) \\
=-\frac{\left|z_{n}\right|^{2}}{N_{0}}-\frac{\left|s^{l}\right|^{2}}{N_{0}}+\frac{2 \operatorname{Re}\left(z_{n}\right) \operatorname{Re}\left(s^{l}\right)+2 \operatorname{Im}\left(z_{n}\right) \operatorname{Im}\left(s^{l}\right)}{N_{0}} \\
+\sum_{j=1}^{\log _{2} L} b_{j} L_{a}\left(b_{j}\right),
\end{array}
$$

where both $\left(-\frac{\left|z_{n}\right|^{2}}{N_{0}}\right)$ and $\left(-\frac{\left|s^{l}\right|^{2}}{N_{0}}=-\frac{1}{N_{0}}\right)$ are invariant over the candidate variable $s_{n}$. In other words, these two constants are ignored by the Approx-Log-MAP algorithm, when the $a$ posteriori symbol probabilities are compared to each other. Therefore, Eq. (9) may be simplified as:

$$
d_{l}=\frac{2 \operatorname{Re}\left(s^{l}\right)}{N_{0}} \operatorname{Re}\left(z_{n}\right)+\frac{2 \operatorname{Im}\left(s^{l}\right)}{N_{0}} \operatorname{Im}\left(z_{n}\right)+\sum_{j=1}^{\log _{2} L} b_{j} L_{a}\left(b_{j}\right)
$$

where both of $\left[\frac{2 \operatorname{Re}\left(s^{l}\right)}{N_{0}}\right]$ and $\left[\frac{2 \operatorname{Im}\left(s^{l}\right)}{N_{0}}\right]$ can be calculated and stored before detection. Therefore, the evaluation of $\left[\frac{2 \operatorname{Re}\left(s^{l}\right)}{N_{0}} \operatorname{Re}\left(z_{n}\right)+\frac{2 \operatorname{Im}\left(s^{l}\right)}{N_{0}} \operatorname{Im}\left(z_{n}\right)\right]$ in Eq. (13) only involves two real-valued multiplications and one real-valued addition. By contrast, evaluating $\left(-\frac{\left|z_{n}-s^{l}\right|^{2}}{N_{0}}\right)$ in Eq. (9) involves a complex-valued subtraction, one complex-valued absolute value calculation, and one real-valued multiplication. In conclusion, we require the Approx-Log-MAP algorithm of Eq. (10) to evaluate the simplified Eq. (13), instead of using the more complex Eq. (9).

\section{Reduced-CompleXity Soft PSK Detection Using THE MAX-LOG-MAP ALGORITHM}

We now take the QPSK constellation of Fig. 1 as an example, where the four a posteriori symbol probabilities of Eq. (13) may be expressed as Eq. (14). It can be seen that the four candidate a posteriori symbol probabilities all contain three parts, i.e. they are $\pm\left[\frac{\sqrt{2}}{N_{0}} \operatorname{Re}\left(z_{n}\right)-\frac{L_{a}\left(b_{2}\right)}{2}\right]$, $\pm\left[\frac{\sqrt{2}}{N_{0}} \operatorname{Im}\left(z_{n}\right)-\frac{L_{a}\left(b_{1}\right)}{2}\right]$ and $\left[\frac{L_{a}\left(b_{1}\right)+L_{a}\left(b_{2}\right)}{2}\right]$. As a result, the maximum distance $d_{\max }$ over these four symbol probabilities $d_{\{1,2,3,4\}}$ may be obtained by taking the absolute value of the first two parts, i.e. we have:

$$
\begin{array}{r}
d_{\max }=\left|\frac{\sqrt{2}}{N_{0}} \operatorname{Re}\left(z_{n}\right)-\frac{L_{a}\left(b_{2}\right)}{2}\right|+\left|\frac{\sqrt{2}}{N_{0}} \operatorname{Im}\left(z_{n}\right)-\frac{L_{a}\left(b_{1}\right)}{2}\right| \\
+\frac{L_{a}\left(b_{1}\right)+L_{a}\left(b_{2}\right)}{2} .
\end{array}
$$

Therefore, instead of evaluating Eq. (13) four times in Eq. (14), Eq. (15) only has to be evaluated once to obtain the maximum distance $d_{\max }$. Since the Max-Log-MAP algorithm in Eq. (11) requires two maximum a posteriori symbol probabilities, we complete the algorithm as:

$$
L_{p}\left(b_{k} \mid \mathbf{Y}\right)=\left\{\begin{array}{ll}
d_{\max }-d_{\max }^{\bar{b}_{k}}, & \text { if } \hat{b}_{k}=1\left(\bar{b}_{k}=0\right) \\
d_{\max }^{\bar{b}_{k}}-d_{\max }, & \text { otherwise }
\end{array},\right.
$$

where $\left\{\bar{b}_{k}\right\}_{k=1}^{\text {BPS }}$ is the negated version of the MAP solution $\left\{\hat{b}_{k}\right\}_{k=1}^{\mathrm{BPS}}$, which is evaluated by:

$$
\begin{aligned}
& \hat{b}_{1}= \begin{cases}1, & \text { if } \frac{\sqrt{2}}{N_{0}} \operatorname{Im}\left(z_{n}\right)-\frac{L_{a}\left(b_{1}\right)}{2}<0, \\
0, & \text { otherwise }\end{cases} \\
& \hat{b}_{2}=\left\{\begin{array}{ll}
1, & \text { if } \frac{\sqrt{2}}{N_{0}} \operatorname{Re}\left(z_{n}\right)-\frac{L_{a}\left(b_{2}\right)}{2}<0 \\
0, & \text { otherwise }
\end{array},\right.
\end{aligned}
$$

which is equivalent to the bit-by-bit based hard QPSK detection of Eq. (7) with the absence of the a priori LLRs.

Based on Eq. (16), we propose the following Max-LogMAP algorithm for soft QPSK detection:

- Step1: The first part of Eq. (15) is evaluated by:

$$
t_{\operatorname{Re}}=\frac{\sqrt{2}}{N_{0}} \operatorname{Re}\left(z_{n}\right)-\frac{L_{a}\left(b_{2}\right)}{2}, \quad d_{\max }^{\operatorname{Re}}=\left|t_{\operatorname{Re}}\right| .
$$

- Step2: The second part of Eq. (15) is given by:

$$
t_{\mathrm{Im}}=\frac{\sqrt{2}}{N_{0}} \operatorname{Im}\left(z_{n}\right)-\frac{L_{a}\left(b_{1}\right)}{2}, \quad d_{\max }^{\operatorname{Im}}=\left|t_{\mathrm{Im}}\right| .
$$

- Step3: The maximum a posteriori symbol probability $d_{\max }$ is found ${ }^{1}$ :

$$
d_{\max }=d_{\max }^{\mathrm{Re}}+d_{\max }^{\mathrm{Im}} .
$$

- Step4: For the first soft output, we have to evaluate:

$$
\begin{aligned}
\hat{b}_{1} & =\left\{\begin{array}{ll}
1, & \text { if } t_{\mathrm{Im}}<0 \\
0, & \text { otherwise }
\end{array},\right. \\
d_{\max }^{\bar{b}_{1}} & =\left\{\begin{array}{ll}
d_{\max }^{\mathrm{Re}}+t_{\mathrm{Im}}, & \text { if } \hat{b}_{1}=1\left(\bar{b}_{1}=0\right), \\
d_{\max }^{\mathrm{Re}}-t_{\mathrm{Im}}, & \text { otherwise }
\end{array},\right.
\end{aligned}
$$

and then $L_{p}\left(b_{1} \mid \mathbf{Y}\right)$ may be obtained by Eq. (16).

- Step5: Similarly, for the second soft output, we have:

$$
\begin{aligned}
\hat{b}_{2} & =\left\{\begin{array}{ll}
1, & \text { if } t_{\operatorname{Re}}<0 \\
0, & \text { otherwise }
\end{array},\right. \\
d_{\max }^{\bar{b}_{2}} & = \begin{cases}t_{\operatorname{Re}}+d_{\max }^{\mathrm{Im}}, & \text { if } \hat{b}_{2}=1\left(\bar{b}_{2}=0\right), \\
-t_{\operatorname{Re}}+d_{\max }^{\mathrm{Im}}, & \text { otherwise }\end{cases}
\end{aligned}
$$

and then $L_{p}\left(b_{2} \mid \mathbf{Y}\right)$ may also be completed by Eq. (16). It can be seen that the channel output $z_{n}$ and the $a$ priori LLRs $\left\{L_{a}\left(b_{j}\right)\right\}_{j=1}^{\text {BPS }}$ has only been processed once in Eqs. (18) and (19). Only testing and combining operations are carried out in the remaining steps.

For our proposed Max-Log-MAP algorithm, the maximum a posteriori symbol probability is no longer obtained by evaluating and comparing all the candidate symbol probabilities. In other words, it is no longer operated on a symbol-by-symbol basis. Since our proposed algorithm is carried out step-bystep, and in each step, only one bit is detected. Therefore we may view our Max-Log-MAP algorithm as a bit-by-bit based reduced-complexity detector.

\footnotetext{
${ }^{1}$ The third part of $\left[\frac{L_{a}\left(b_{1}\right)+L_{a}\left(b_{2}\right)}{2}\right]$ is a common constant for $d_{\max }$ and $d_{\max }^{\bar{b}_{k}}$, and therefore it can be omitted.
} 


$$
\begin{array}{ll}
d_{1}=\frac{\sqrt{2}}{N_{0}} \operatorname{Re}\left(z_{n}\right)+\frac{\sqrt{2}}{N_{0}} \operatorname{Im}\left(z_{n}\right) & =\left[\frac{\sqrt{2}}{N_{0}} \operatorname{Re}\left(z_{n}\right)-\frac{L_{a}\left(b_{2}\right)}{2}\right]+\left[\frac{\sqrt{2}}{N_{0}} \operatorname{Im}\left(z_{n}\right)-\frac{L_{a}\left(b_{1}\right)}{2}\right]+\frac{L_{a}\left(b_{1}\right)+L_{a}\left(b_{2}\right)}{2}, \\
d_{2}=-\frac{\sqrt{2}}{N_{0}} \operatorname{Re}\left(z_{n}\right)+\frac{\sqrt{2}}{N_{0}} \operatorname{Im}\left(z_{n}\right)+L_{a}\left(b_{2}\right) & =\left[-\frac{\sqrt{2}}{N_{0}} \operatorname{Re}\left(z_{n}\right)+\frac{L_{a}\left(b_{2}\right)}{2}\right]+\left[\frac{\sqrt{2}}{N_{0}} \operatorname{Im}\left(z_{n}\right)-\frac{L_{a}\left(b_{1}\right)}{2}\right]+\frac{L_{a}\left(b_{1}\right)+L_{a}\left(b_{2}\right)}{2}, \\
d_{3}=\frac{\sqrt{2}}{N_{0}} \operatorname{Re}\left(z_{n}\right)-\frac{\sqrt{2}}{N_{0}} \operatorname{Im}\left(z_{n}\right)+L_{a}\left(b_{1}\right) & =\left[\frac{\sqrt{2}}{N_{0}} \operatorname{Re}\left(z_{n}\right)-\frac{L_{a}\left(b_{2}\right)}{2}\right]+\left[-\frac{\sqrt{2}}{N_{0}} \operatorname{Im}\left(z_{n}\right)+\frac{L_{a}\left(b_{1}\right)}{2}\right]+\frac{L_{a}\left(b_{1}\right)+L_{a}\left(b_{2}\right)}{2}, \\
d_{4}=-\frac{\sqrt{2}}{N_{0}} \operatorname{Re}\left(z_{n}\right)-\frac{\sqrt{2}}{N_{0}} \operatorname{Im}\left(z_{n}\right)+L_{a}\left(b_{1}\right)+L_{a}\left(b_{2}\right) & =\left[-\frac{\sqrt{2}}{N_{0}} \operatorname{Re}\left(z_{n}\right)+\frac{L_{a}\left(b_{2}\right)}{2}\right]+\left[-\frac{\sqrt{2}}{N_{0}} \operatorname{Im}\left(z_{n}\right)+\frac{L_{a}\left(b_{1}\right)}{2}\right]+\frac{L_{a}\left(b_{1}\right)+L_{a}\left(b_{2}\right)}{2}
\end{array}
$$

TABLE I

THE MAXIMUM $a$ posteriori SYMBOL PROBABILITY CALCULATION FOR DIFFERENT $L$-PSK SCHEMES.

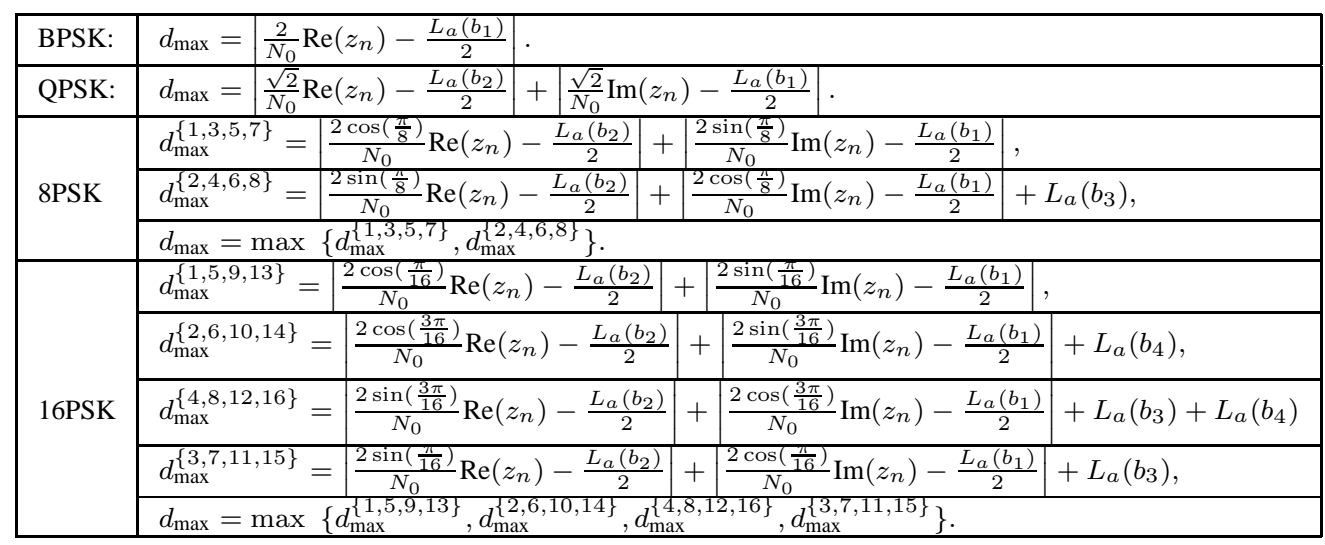

TABLE II

LOOKUP TABLE FOR COMPUTATIONAL COMPLEXITY

\begin{tabular}{|l|c|}
\hline $\begin{array}{l}\text { real addition/multiplication, comparison, } \\
\text { real absolute value calculation }\end{array}$ & +1 \\
\hline complex addtion & +2 \\
\hline complex multiplication & +6 \\
\hline complex absolute value calculation & +3 \\
\hline $\begin{array}{l}\text { complex matrix addition } \\
\text { (both matrices have } u \times v \text { elements) }\end{array}$ & $+2 u v$ \\
\hline $\begin{array}{l}\text { complex matrix multiplication } \\
\text { (the matrices have } u \times v \text { and } v \times w \text { elements) }\end{array}$ & $+(8 v-2) u w$ \\
\hline $\begin{array}{l}\text { complex matrix norm calculation } \\
\text { (the matrix has } u \times v \text { elements) }\end{array}$ & $+(4 u v-1)$ \\
\hline
\end{tabular}

We summarize the maximum a posteriori symbol probability calculation for different $L$-PSK schemes in Table I. The complete reduced-complexity Max-Log-MAP algorithm conceived for detecting different $L$-PSK constellations may be carried out in the same way as that designed for QPSK.

\section{Performance Results}

We provide our simulation results in this section. First of all, we note that our proposed Approx-Log-MAP algorithm using Eq. (13) has the same detection capability as the original Approx-Log-MAP algorithm using Eq. (9). We have arranged both detectors to detect the same channel output associated with the same a priori LLRs, and found that they always produce exactly the same a posteriori LLR values. Secondly, we have also verified that our proposed Max-LogMAP algorithm designed for soft PSK detection introduced in Section V is completely equivalent to the conventional MaxLog-MAP algorithm using Eq. (9).

The complexity comparison between the conventional soft PSK detectors and our proposed algorithms is portrayed in Fig. 2. We quantify the complexity in terms of the total number of floating point calculations required for evaluating a single PSK symbol, and we provide a summary of our complexity results in Table II. Fig. 2 shows that by using

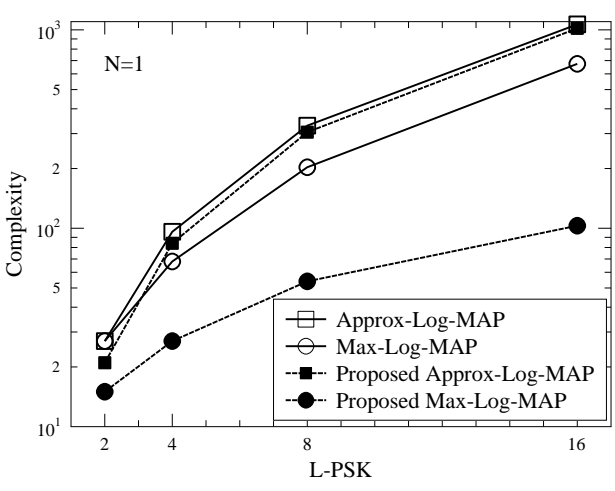

Fig. 2. Complexity comparison between the conventional soft detectors introduced in Section III, and the proposed reduced-complexity soft detectors.

the simplified Eq. (13) instead of directly using Eq. (9), our proposed Approx-Log-MAP algorithm exhibits a reduced complexity. This complexity reduction may become more beneficial, once turbo detection is invoked. Furthermore, it is also shown in Fig. 2 that our proposed Max-Log-MAP algorithm introduced in Section V has a substantially lower complexity, than the conventional Max-Log-MAP algorithm introduced in Section III. This is because our proposed Max-Log-MAP algorithm produces each single soft bit output without estimating all the candidate a posteriori symbol probabilities.

The performance of our proposed soft detectors is characterized with the aid of EXIT charts [11] in Fig. 3, which comfirms that the employment of the Max-Log-MAP algorithm leads to a slight performance loss for soft $L$-PSK detection, when we have $(L>4)^{2}$. Fig. 4 further portrays the BER performance of our proposed soft detectors, when

${ }^{2}$ For soft BPSK/QPSK detection, the difference between the optimum $a$ posteriori symbol probability and the non-optimum ones is beyond the scope defined by the lookup table in [4]. Therefore the Approx-Log-MAP algorithm and the Max-Log-MAP algorithm designed for soft BPSK/QPSK detection always produce the same result. 


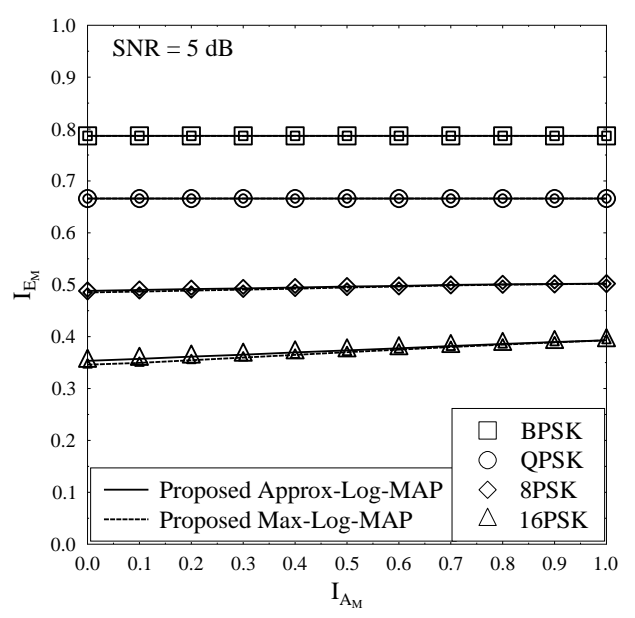

Fig. 3. EXIT charts of BPSK, QPSK, 8PSK as well as 16PSK.

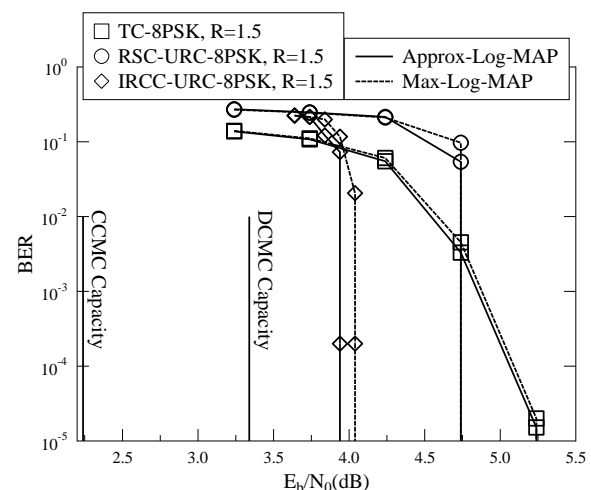

Fig. 4. BER performance of the RSC-URC coded, TC coded, and IRCCURC coded 8PSK.

they are applied to various coded systems. The TC schematic and the three-component serially concatenated IRCC-URCPSK schematic may be found in [5] and [13], respectively. The ergodic Continuous-input Continuous-output Memoryless Channel's (CCMC) capacity and the achievable Discrete-input Continuous-output Memoryless Channel's (DCMC) capacity are calculated according to [14], [6]. It is evidenced by Fig. 4 that a near-capacity performance may be achieved by the proposed reduced-complexity soft 8PSK detectors with the aid of channel coding and turbo detection. However, a slight performance loss is imposed by using the Max-Log-MAP algorithm, as seen in Fig. 4. Nonetheless, this loss is limited to $0.2 \mathrm{~dB}$ for the IRCC-URC-8PSK scheme. Fig. 5 shows a further performance comparison of QPSK, 8PSK and 16PSK, when they are detected by the proposed reduced-complexity detectors, which are combined with the IRCC and URC coded schemes. It can be seen that a near-capacity performance is achieved by all the three different modulation schemes.

\section{CONCLUSIONS}

A new method of reducing the complexity of the softdecision aided PSK detection was proposed. We first simplified the $a$ posteriori symbol probability equation evaluated by the Approx-Log-MAP algorithm. We then conceived a new MaxLog-MAP algorithm for PSK detection, where the maximum a posteriori symbol probability may be obtained without

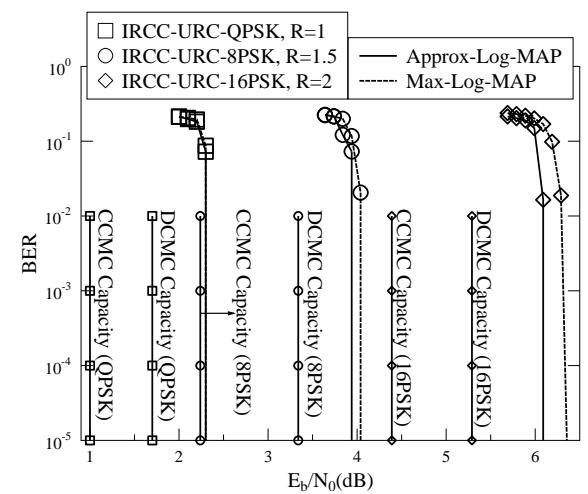

Fig. 5. BER performance of the IRCC and URC coded QPSK, 8PSK and 16PSK.

estimating and comparing all the candidate symbol probabilities. We applied the proposed soft detection algorithms to both different modulation schemes and to a variety of coded systems. Our simulation results comfirmed that the proposed Approx-Log-MAP algorithm and Max-Log-MAP algorithm are capable of achieving a near-capacity performance at a reduced complexity in channel coding aided turbo detection assisted communication systems.

\section{REFERENCES}

[1] L. Bahl, J. Cocke, F. Jelinek, and J. Raviv, "Optimal decoding of linear codes for minimizing symbol error rate," IEEE Transactions on Information Theory, vol. 20, pp. 284 - 287, Mar. 1974.

[2] J. Hagenauer and P. Hoeher, "A Viterbi algorithm with soft-decision outputs and its applications," in IEEE Global Telecommunications Conference (GLOBECOM'89), pp. 1680 -1686 vol.3, Nov. 1989.

[3] W. Koch and A. Baier, "Optimum and sub-optimum detection of coded data disturbed by time-varying intersymbol interference," in IEEE Global Telecommunications Conference (GLOBECOM'90), pp. $1679-$ 1684 vol.3, Dec. 1990.

[4] P. Robertson, E. Villebrun, and P. Hoeher, "A comparison of optimal and sub-optimal MAP decoding algorithms operating in the log domain," in IEEE International Conference on Communications (ICC'95), vol. 2, pp. $1009-1013$ vol.2, June 1995.

[5] C. Berrou and A. Glavieux, "Near optimum error correcting coding and decoding: Turbo-codes," IEEE Transactions on Communications, vol. 44, pp. $1261-1271$, Oct. 1996.

[6] S. X. Ng and L. Hanzo, "On the MIMO channel capacity of multidimensional signal sets," IEEE Transactions on Vehicular Technology, vol. 55, pp. 528 - 536, Mar. 2006.

[7] L. Hanzo, L. Hanzo, O. Alamri, M. El-Hajjar, and N. Wu, Near-Capacity Multi-Functional MIMO Systems(Sphere-Packing, Iterative Detection and Cooperation). John Wiley \& Sons, May 2009.

[8] L. Hanzo, S. X. Ng, W. T. Webb, and T. Keller, Quadrature Amplitude Modulation: From Basics to Adaptive Trellis-Coded, Turbo-Equalised and Space-Time Coded OFDM, CDMA and MC-CDMA Systems, 3rd Edition. John Wiley \& Sons, Sept. 2004.

[9] L. Hanzo, T. Liew, B. Yeap, R. Tee, and S. Ng, Turbo Coding, Turbo Equalisation and Space-Time Coding(EXIT-Chart-Aided Near-Capacity Designs for Wireless Channels). 2011.

[10] S. Ten Brink, J. Speidel, and R.-H. Han, "Iterative demapping for QPSK modulation," Electronics Letters, vol. 34, pp. 1459 -1460, July 1998.

[11] S. Ten Brink, "Convergence behavior of iteratively decoded parallel concatenated codes," IEEE Transactions on Communications, vol. 49, pp. $1727-1737$, Oct. 2001

[12] H. Tullberg and P. Siegel, "Serial concatenated TCM with an inner accumulate code-Part I: maximum-likelihood analysis," IEEE Transactions on Communications, vol. 53, pp. 64 - 73, Jan. 2005.

[13] M. Tuchler, "Design of serially concatenated systems depending on the block length," IEEE Transactions on Communications, vol. 52, pp. 209 - 218, Feb. 2004.

[14] C. Shannon, "A mathematical theory of communication," Bell Systems Technical Journal, vol. 27, pp. 379-423, June 1948. 